\title{
Caracterización clínica de los casos de dengue hospitalizados en la E.S.E. Hospital “Antonio Roldán Betancur”, Apartadó, Antioquia, Colombia, 2000
}

\author{
Margarita Arboleda ', Mónica Campuzano ${ }^{2}$, Berta Nelly Restrepo ${ }^{1}$, Gladys Cartagena ${ }^{2}$ \\ 1 Instituto Colombiano de Medicina Tropical - CES, Apartadó, Antioquia, Colombia. \\ 2 Empresa Social del Estado Hospital Antonio Roldán Betancur, Apartadó, Antioquia, Colombia.
}

Introducción. Desde 1998 se vienen notificando casos de dengue hemorrágico en el municipio de Apartadó y actualmente el dengue se considera endemo-epidémico.

Objetivo. Describir el comportamiento clínico del dengue en pacientes hospitalizados por esta causa en la Empresa Social del Estado Hospital Antonio Roldán Betancur de Apartadó en el año 2000.

Materiales y métodos. Estudio descriptivo retrospectivo de revisión de historias clínicas de pacientes hospitalizados por dengue.

Resultados. Se hospitalizaron 45 casos con diagnóstico serológico de dengue. Tres (6,7\%) pacientes con dengue clásico, $22(48,9 \%)$ con dengue con manifestaciones hemorrágicas y $20(44,4 \%)$ con dengue hemorrágico. La edad osciló entre 4 meses y 68 años con un promedio de 22,1 años. La mayoría procedía del área urbana $(82,8 \%)$. Las manifestaciones clínicas más frecuentes fueron: fiebre (100\%), mialgias y artralgias $(71,1 \%)$, cefalea $(70,5 \%)$ y dolor abdominal $(67,5 \%)$. Las manifestaciones hemorrágicas más frecuentes fueron petequias $(60 \%)$, hematuria $(31,7 \%)$, equimosis en sitios de venopunción $(24,4 \%)$, melenas $(22,2 \%)$, hematemesis $(15,5 \%)$ y hemorragias vaginales anormales (15,5\%). El recuento de plaquetas osciló entre 11.000 y $186.000 / \mathrm{mm}^{3}$, con un promedio de 48.688. Los hematocritos oscilaron entre 26 y $59 \%$, con un promedio de $41,5 \%$. Fallecieron 4 (8,8\%) pacientes, tres de éstos menores de un año. En el $40 \%$ de los casos se hicieron otros diagnósticos simultáneamente, siendo malaria la coinfección más frecuente, con $17,7 \%(8 / 45)$ de los casos.

Conclusión. En este estudio las personas en edad productiva tuvieron mayor riesgo de sufrir dengue y la población menor de un año fue la de mayor riesgo de letalidad; existe, además, la posibilidad de coinfección con otras enfermedades, particularmente malaria.

Palabras clave: dengue, fiebre dengue hemorrágica, comorbilidad.

The clinical behavior of dengue in patients hospitalized in the Antonio Roldán Betancur Hospital of Apartadó, Antioquia, 2000.

Introduction. Dengue has exhibited an epidemic behaviour in Apartadó since 1998.

Objective. To describe the clinical behavior of dengue in patients hospitalized with this diagnosis in the Antonio Roldán Betancur Hospital of Apartadó in the year 2000.

Materials and methods. Descriptive retrospective study with variables obtained from clinical records of patients hospitalized with dengue in the Antonio Roldán Betancur Hospital of Apartadó. Results. 45 patients with the diagnosis of dengue were hospitalized during the study period, all of them with $\operatorname{lgM}$ antibodies against dengue virus. Three (6.7\%) patients were classified as classic dengue fever, $22(48.9 \%)$ as dengue fever with haemorrhagic manifestations, and 20 patients $(44.4 \%)$ were classified as dengue haemorrhagic fever. Patient age ranged from 4 months to 68 years, with a mean of 22.1 years. The great majority of patients $(82.8 \%)$ came from urban areas.

The most frequent clinical manifestations were fever (100\%), myalgia (71.1\%), joint pain (71.1\%), headache $(70 \%)$ and abdominal pain $(67.5 \%)$. The most frequent haemorrhagic manifestations were petechiae $(60 \%)$, hematuria $(31.7 \%)$, ecchymoses on venipuncture sites $(24.4 \%)$, gastric bleeding (22.2\%) and abnormal vaginal bleeding (15.5\%). Platelet count ranged from 11.000 
to $186.000 / \mathrm{mm}^{3}$, with a mean of 48.688 . Hematocrit ranged from $26 \%$ to $59 \%$ with a mean of $41.5 \%$. Four of the 45 patients died. $40 \%$ of patients had other co-morbidities; coinfection with malaria was the most frequent amongst them (8 patients, 17.7\%).

Conclusion. Two remarkable findings of our descriptive retrospective study were the high mortality of dengue in our population (8.8\%) 4/45, specially in children under 1 year of age (3/4) and the high incidence of simultaneous infection with malaria parasites.

Keywords: dengue, dengue haemorrhagic fever, comorbidity.

El dengue es una enfermedad febril aguda producida por el virus del dengue, el cual pertenece a la familia Flaviviridae, género Flavivirus y cuenta con cuatro serotipos (Den-1, Den-2, Den-3 y Den-4), que son transmitidos por mosquitos del género Aedes (aegypti y albopictus), siendo $A$. aegypti el de mayor importancia en el continente americano, en el que ha circulado ampliamente después de reinfestar casi todos los países a partir de los años 60 (1). El dengue es la arbovirosis humana con mayores implicaciones desde el punto de vista de salud pública por la alta morbilidad y mortalidad que genera.

La infección por el virus del dengue causa un amplio espectro de la enfermedad que va desde formas inaparentes a formas graves y fatales como son la enfermedad hemorrágica y el síndrome del choque del dengue (DH/SCD), pasando por la forma benigna, denominada dengue clásico (DC) (2). La infección confiere inmunidad de por vida para cada serotipo (1).

El comportamiento del dengue en Colombia ha sido endémico con brotes epidémicos. Alrededor de 20 millones de personas están bajo riesgo de sufrir la enfermedad. En 1995 se notificaron 12.430 casos de dengue y en el 2002, 81.831 casos, lo que implica un riesgo 6,5 veces mayor en el presente año con respecto a 1995. En el año 2003, el total de casos fue de 58.309 (3). Desde la detección del primer caso de dengue hemorrágico en 1989 , se ha observado una tendencia al

\footnotetext{
Correspondencia:

Margarita Arboleda,

Instituto Colombiano de Medicina Tropical-CES, ESE Hospital ARB, Barrio Chinita, Apartadó, Antioquia, Colombia. Tel. (574) 8281708 - 8285606, fax: (574) 8281708 8285606

mtropi@edatel.net.co
}

Recibido: 14/03/06; aceptado: 19/05/06 ascenso, pasando de 1,4 casos por 100.000 habitantes en 1994 a 5,17 casos por 100.000 en 1998, año en el que fueron notificados 5.276 casos. En el 2003 se notificaron 5.026 casos de dengue hemorrágico (3).

En el departamento de Antioquia el promedio anual de casos de dengue clásico en los últimos cinco años (1999-2003) fue de 3.065 y de dengue hemorrágico, 93. (4). El año con mayor número de enfermos fue 1998, con 13.137 casos de dengue clásico, para una proporción de incidencia de 245,8 por 100.000 habitantes y de 222 casos de dengue hemorrágico para una proporción de incidencia de 4,27 por cien mil habitantes (5). El dengue se considera endemo-epidémico en la región de Urabá. En el quinquenio 1999-2003 se reportó un promedio de 347 casos de dengue clásico y 45 casos de dengue hemorrágico, con una proporción de incidencia para el año 2003 de 53,8 casos de dengue clásico por 100.000 habitantes y de 8,2 casos de dengue hemorrágico por 100.000 habitantes en el mismo año, casos éstos que sólo se han notificado desde 1998 (4). En un estudio realizado en nueve municipios del Urabá antioqueño se encontró una seroprevalencia de anticuerpos IgG contra el virus del dengue del $55,7 \%$ (6). Así mismo, los índices de infestación larvaria y de Breteau, superiores al $35 \%$ en el área urbana, colocan al municipio de Apartadó como de alto riesgo para infección por virus del dengue (Dirección Local de Salud, Municipio de Apartadó. Registros de Vigilancia Epidemiológica y Entomológica, año 2000).

La Empresa Social del Estado Hospital "Antonio Roldán Betancur", localizada en Apartadó, Antioquia, es una institución de segundo nivel y es el principal centro de atención de pacientes con dengue de la zona de Urabá. El presente estudio tuvo como objetivo describir el comportamiento clínico del dengue en pacientes 
hospitalizados en esta institución durante el año 2000, caracterizando su forma de presentación, hallazgos de laboratorio, coinfecciones y letalidad, con el fin de mejorar el conocimiento epidemiológico del dengue en nuestra región y, en consecuencia, aportar elementos que orienten las medidas de intervención.

\section{Materiales y métodos}

Se trata de un estudio descriptivo retrospectivo cuya población de estudio correspondió a la totalidad de casos confirmados de dengue que fueron hospitalizados en la Empresa Social del Estado Hospital "Antonio Roldán Betancur", durante el año 2000.

El diagnóstico de dengue se hizo mediante la detección de anticuerpos IgM contra el virus del dengue a través de la prueba comercial UMELISA® dengue IgM (Centro de Inmunoensayo, Instituto Pedro Kourí, La Habana, Cuba, prueba estandarizada para el diagnóstico de dengue en la red de laboratorios de salud pública del país), y se realizó en el Laboratorio Departamental de Salud Pública de Antioquia. Los exámenes de laboratorio e imaginología se realizaron con métodos convencionales en la Empresa Social del Estado Hospital "Antonio Roldán Betancur".

Se revisaron las historias clínicas de los pacientes hospitalizados por dengue y se obtuvieron los datos correspondientes a las variables demográficas, clínicas, exámenes de laboratorio, evolución y coinfecciones. Los casos de dengue hemorrágico fueron clasificados de acuerdo con los criterios de la Organización Mundial de la Salud (OMS) para fiebre hemorrágica dengue; los pacientes con criterios de choque circulatorio según esta clasificación fueron catalogados como síndrome de choque por dengue (SCD) (7). Los casos que no llenaban estos criterios fueron clasificados como dengue con manifestaciones hemorrágicas $(\mathrm{DCMH})$ o dengue clásico según presentaran o no hemorragias clínicamente evidentes. Las manifestaciones hemorrágicas tales como hematuria y melenas fueron confirmadas por examen microscópico de orina y búsqueda de sangre oculta en heces.

La información obtenida se ingresó a una base de datos elaborada en Microsoft Excel 97 (Microsoft
Corp., Redmond, WA). El análisis se hizo mediante el programa Epi-Info versión 6.04c, utilizando las medidas generales estandarizadas para estudios descriptivos.

\section{Resultados}

En el año 2000 se hospitalizaron 57 pacientes en la Empresa Social del Estado Hospital "Antonio Roldán Betancur" de Apartadó con cuadros febriles agudos cuya sospecha diagnóstica fue dengue. De éstos, 12 casos quedaron catalogados como casos probables y 45 tuvieron anticuerpos $\operatorname{lgM}$ positivos contra dengue. El 6,7\% (3/45) de los casos confirmados correspondieron a dengue clásico, el 48,9\% (22/45) a dengue con manifestaciones hemorrágicas y el $44,4 \%(20 / 45)$ restante a casos de dengue hemorrágico; de los casos de dengue hemorrágico, el 25\% (5/20) satisfacía los criterios de síndrome de choque por dengue. En 25 de los 45 casos hospitalizados $(55,5 \%)$ se registraron diferencias de hematocrito menores a $20 \%$ y en 18 de estos 25 casos la diferencia fue mayor de $10 \%$.

La edad de los pacientes osciló entre 5 meses y 68 años, con un promedio de 22,1 años. En la figura 1 se muestra la distribución de los pacientes hospitalizados por grupos de edad.

El $55,6 \%$ de los casos correspondió al género femenino (25/45) y el $44,4 \%$ al género masculino (ji al cuadrado $=1,1 ; p=0,29$ ). Aproximadamente la mitad de los casos (22/45: 48,9\%) procedía del municipio de Apartadó y los demás (23/45) de municipios vecinos (Turbo: 10, Carepa: 10, Chigorodó: 1, Mutatá: 1 y San Pedro de Urabá: 1). Sólo en 29 casos se obtuvo información sobre

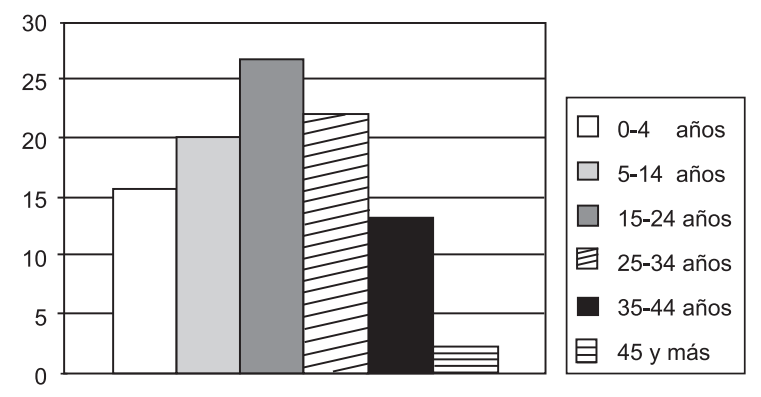

Figura 1. Pacientes hospitalizados por dengue según grupo de edad. ESE Hospital Antonio Roldán Betancur, Apartadó, 2000. 
procedencia, correspondiendo $82,2 \%$ de los casos al área urbana y $17,2 \%$, al área rural.

Los principales síntomas generales referidos por los pacientes fueron fiebre, mialgias, artralgias y cefalea (cuadro 1).

El dolor abdominal fue un hallazgo más frecuente en los casos de dengue hemorrágico $(81,2 \%)$ que en los casos de dengue con manifestaciones hemorrágicas (54,5\%). Esta diferencia no fue estadísticamente significativa (ji al cuadrado $=2,86$; $p=0,09$ ).

Los sitios de sangrado más comunes fueron la piel, el tracto urinario y el tracto gastrointestinal, tal como se observa en el cuadro 2. Otra manifestación frecuente fue la hemorragia vaginal anormal que se presentó en $7(28 \%)$ de las 25 mujeres hospitalizadas, quienes, además, siempre presentaron alguna otra manifestación hemorrágica (hematuria, petequias, epistaxis, gingivorragia, equimosis en sitios de venopunción y melenas). No se observaron diferencias entre los hallazgos clínicos encontrados en los pacientes con dengue con manifestaciones hemorrágicas y los pacientes con dengue hemorrágico.

Se realizó prueba del torniquete a 27 de los 45 pacientes, siendo ésta positiva en el $63 \%$ (17/27) de los casos. Se solicitó ecografía abdominal para 10 pacientes que relataron dolor abdominal y en siete de éstos se detectaron hallazgos compatibles con serositis: un paciente con derrame pleural,

Cuadro 1. Pacientes hospitalizados por dengue según frecuencia de síntomas generales. ESE Hospital Antonio Roldán Betancur, Apartadó, 2000.

\begin{tabular}{lcc}
\hline Síntomas & Número & $\%$ \\
\hline Fiebre & 45 & 100 \\
Mialgias y artralgias & 32 & 71,1 \\
Cefalea & 31 & 70,5 \\
Dolor abdominal & 27 & 67,5 \\
Vómito & 21 & 46,7 \\
Dolor retrocular & 20 & 44,4 \\
Escalofrío & 17 & 38,6 \\
Diarrea & 14 & 31,1 \\
Erupción cutánea & 8 & 17,7 \\
Hepatomegalia & 8 & 17,7 \\
Esplenomegalia & 4 & 8,8 \\
\hline
\end{tabular}

Cuadro 2. Pacientes hospitalizados por dengue según frecuencia de manifestaciones hemorrágicas. ESE Hospital Antonio Roldán Betancur, Apartadó, 2000.

\begin{tabular}{lcc}
\hline Síntomas & Número & Porcentaje \\
Petequias & 27 & 60,0 \\
Hematuria & 13 & 31,7 \\
Equimosis & 11 & 24,4 \\
Melenas & 10 & 22,2 \\
Hematemesis & 7 & 15,5 \\
Hemorragia vaginal & 7 & $28,0^{*}$ \\
Epistaxis & 6 & 13,3 \\
Gingivorragia & 4 & 8,9 \\
\hline
\end{tabular}

${ }^{*} \mathrm{n}=25$ mujeres

dos con ascitis, y tres que presentaron simultáneamente derrame pleural y ascitis.

De los cinco pacientes con criterios de síndrome de choque por dengue, tres fallecieron, uno se remitió a un hospital de tercer nivel y otro fue tratado en la institución en unidad de cuidados críticos. Todos los pacientes con síndrome de choque por dengue presentaron manifestaciones hemorrágicas, predominando los sangrados de tipo gastrointestinal.

El valor mínimo plaquetario fue de $11.000 / \mathrm{mm}^{3}$, el máximo de $186.000 / \mathrm{mm}^{3}$ y el promedio de $48.688 / \mathrm{mm}^{3}$. Los recuentos menores de $50.000 /$ $\mathrm{mm}^{3}$ fueron ligeramente más frecuentes en los casos de dengue hemorrágico (15/20) que en los de dengue con manifestaciones hemorrágicas (13/ 22). Esta diferencia no fue estadísticamente significativa $(p=0,27)$. Solo hubo dos casos con recuentos plaquetarios mayores de 150.000 plaquetas $/ \mathrm{mm}^{3}$ y éstos correspondieron a pacientes clasificados con dengue con manifestaciones hemorrágicas.

En el 40\% (18/45) de los casos se documentaron simultáneamente otros diagnósticos: malaria, ocho casos: seis por Plasmodium vivax y dos por Plasmodium falciparum; sepsis urinaria, cuatro casos; pielonefritis, dos casos; bronconeumonía, dos casos; intoxicación alimentaria, un caso, y aborto e infección por citomegalovirus, un caso.

Se presentaron cinco casos de dengue en pacientes obstétricas, una de las cuales abortó en el tercer mes de gestación. Esta paciente tenía también infección por citomegalovirus comprobado mediante estudio serológico a la 
gestante -anticuerpos $\lg \mathrm{M}-\mathrm{y}$ estudio anatomopatológico de restos placentarios realizado en el Laboratorio Departamental de Salud Pública de Antioquia.

Fallecieron cuatro de los 45 pacientes hospitalizados $(8,8 \%)$, tres por choque circulatorio y uno con bronconeumonía asociada. Tres de los fallecidos eran menores de un año (5, 9 y 11 meses) y el otro tenía un año cumplido (16 meses).

\section{Discusión}

De los 45 casos hospitalizados por dengue en la Empresa Social del Estado Hospital "Antonio Roldán Betancur" en el año 2000, aproximadamente la mitad (48,9\%) se clasificó como dengue con manifestaciones hemorrágicas. Probablemente algunos de éstos fueron casos de dengue hemorrágico en los que no fue posible documentar la totalidad de criterios establecidos por la OMS (7), particularmente el criterio de hemoconcentración, es decir, casos en los cuales la diferencia entre hematocritos no fue mayor del $20 \%$. En su trabajo sobre dengue hemorrágico en niños, Méndez y González (8) plantean, al igual que otros autores, que la hemoconcentración es el criterio más difícil de establecer en la práctica clínica, y encontraron aumento del hematocrito en más de $10 \%$, pero menor de $20 \%$, en 60 de 118 pacientes en quienes no se pudo demostrar fuga vascular, pero que sí cumplían los demás criterios para fiebre hemorrágica por dengue.

Llama la atención que la mayor parte de los grupos de edad afectados son menores de 34 años, predominando el grupo de 15 a 24 años, situación que ha sido descrita por otros autores $(9,10)$, posiblemente por una mayor exposición al vector. El predominio del sexo femenino en este estudio, $1,5: 1$, coincide con lo reportado por algunos autores $(6,11,12)$ y difiere en otros en los que el más afectado es el sexo masculino $(9,13)$, o en otros en que ambos sexos están afectados en forma semejante (10), indicando diferencias por sexo en la exposición al vector.

Se identificó que el dengue es una arbovirosis de predominio urbano, tal como se describe en la literatura (2), pero se reportaron algunos casos procedentes del área rural (17,2\%). Este último hallazgo se ha descrito en otros lugares del mundo como la India, donde hay reportes de casos de dengue en zonas rurales y periurbanas con presencia de infestación de $A$. aegypti (14-16). Igualmente, en Indonesia se han documentado brotes en zonas rurales (17). En Perú se han detectado prevalencias de anticuerpos semejantes entre la población rural y urbana (67 y $66 \%$, respectivamente) (17).

Las manifestaciones clínicas más frecuentes fueron fiebre, mialgias y artralgias y cefalea, lo que coincide con lo reportado en la literatura $(1,2$, 18,19). El dolor abdominal se considera un signo de alarma en pacientes con dengue hemorrágico $(1,7,20)$. En el presente estudio este síntoma fue 1,5 veces más frecuente en los casos de dengue hemorrágico que en los de dengue con manifestaciones hemorrágicas. Conforme se mencionó anteriormente, pudo ocurrir que varios casos clasificados como dengue con manifestaciones hemorrágicas fueran probablemente casos de dengue hemorrágico que se acompañaron de dolor abdominal en los cuales no fue posible establecer todos los criterios para catalogarlos como tales.

Se destaca en el presente estudio la alta frecuencia de síntomas gastrointestinales caracterizados por vómito y diarrea (46,7 y 31,1\%, respectivamente). En Cuba, Vargas y cols. (9) reportan frecuencias menores de estos síntomas en pacientes pediátricos con dengue, 10,3 y 9,0\%, respectivamente. También en Cuba, Martínez y cols. (18) reportan $25 \%$ de pacientes con diarrea y $50 \%$ con vómitos, coincidiendo este último dato con los hallazgos del presente estudio. En una serie de 114 pacientes con dengue hemorrágico, Guzmán y cols. (21) encontraron una frecuencia de 74 y $23 \%$, respectivamente. En Colombia, Convers y cols. (20) relatan presencia de vómito en $73 \%$ de los casos de dengue clásico y en $83 \%$ de los casos de dengue hemorrágico.

Se ha descrito la hepatomegalia como un hallazgo frecuente que suele ocurrir en el $90 \%$ de los casos de dengue hemorrágico en niños y en alrededor del $60 \%$ en los adultos entre el tercero y cuarto día de enfermedad (7). En el presente estudio la 
hepatomegalia no fue un hallazgo frecuente registrándose en el $17,7 \%$ de los casos, aunque sí fue mayor en los casos de dengue hemorrágico que en los casos de dengue con manifestaciones hemorrágicas (6/20 vs. 2/22), pero la diferencia no fue estadísticamente significativa (ji al cuadrado $=1,07$; prueba de Fisher: $p=0,15$ a 1 cola; $p=0,25$ a 2 colas).

La prueba del torniquete fue positiva en 17 de los 27 (63\%) pacientes evaluados con este procedimiento: seis con dengue hemorrágico y 11 con dengue con manifestaciones hemorrágicas. En los pacientes estudiados en Cali, la prueba del torniquete fue positiva con mayor frecuencia en los casos con dengue hemorrágico que en los casos de dengue con manifestaciones hemorrágicas (22).

Las manifestaciones hemorrágicas más frecuentes en los pacientes del presente estudio fueron las de piel, petequias $(60 \%)$ y equimosis en sitios de venopunción $(24,4 \%)$, lo cual coincide con lo descrito por algunos autores en la literatura $(1,8,18,21,23)$. En el presente estudio la hematuria se presentó en el 31,7\% (13/45) de los pacientes, correspondiendo a $35 \%$ de los pacientes con dengue hemorrágico y $33 \%$ de los pacientes con dengue con manifestaciones hemorrágicas. Este signo no ha sido una manifestación hemorrágica frecuente en los estudios de dengue hemorrágico de Cuba $(9,18,21)$. De estos pacientes con hematuria, en cuatro se diagnosticaron simultáneamente otros eventos: un paciente con malaria por $P$. vivax, dos pacientes con infección urinaria y una paciente que abortó y tenía infección por citomegalovirus. La hematuria es una manifestación clínica que normalmente puede presentarse en los cuadros descritos y no necesariamente es una manifestación exclusiva de la infección por dengue. La trombocitopenia y la anemia hemolítica se han asociado en algunos casos con la infección por citomegalovirus $(24,25)$, con sangrado y manifestaciones purpúricas. Así mismo, la malaria puede acompañarse de hematuria, sea por la anemia hemolítica, por la misma trombocitopenia, o por el daño glomerular que se presenta secundario a la insuficiencia renal $(26,27)$.
Se destaca en el presente estudio la alta proporción de hemorragia vaginal anormal $(15,5 \%)$, hallazgo que es comparable al descrito por Guzmán y cols. en $23 \%$ de mujeres con dengue hemorrágico (21), y por Restrepo y cols., con 37,5 y $17,9 \%$ en dos cohortes de gestantes con dengue (28).

En 10 casos de los que refirieron dolor abdominal se solicitó estudio ecográfico demostrándose presencia de líquido libre en cavidad abdominal, o derrame pleural en siete de éstos (70\%); a su vez, en tres de los 10 casos con ecografía, el derrame seroso fue el único criterio de hemoconcentración, pues no hubo diferencias de hematocritos mayores del $20 \%$. Esta observación corrobora la sensibilidad de la ecografía para detectar la presencia de líquido en el tercer espacio, que es otra forma de documentar la hemoconcentración. Salgado y cols (29) encontraron derrames serosos (pleurales, peritoneales y pericárdicos) en el $40 \%$ de los casos estudiados en la epidemia de dengue hemorrágico en Neiva, relacionándolos con la gravedad de la fuga vascular, y siendo responsables del $30 \%$ de los pacientes con choque por dengue hemorrágico. En Cuba se ha reportado un $37,5 \%$ de casos de dengue hemorrágico con ascitis y $12,5 \%$ con derrame pleural (18).

El recuento plaquetario más bajo observado en los pacientes del presente estudio fue de 11.000 y el recuento promedio fue de 48.688 plaquetas. Los recuentos menores de 50.000 plaquetas fueron ligeramente más frecuentes en los casos de dengue hemorrágico (15/20) que en los de dengue con manifestaciones hemorrágicas (13/22). En el estudio de Neiva se reportan recuentos plaquetarios similares, con trombocitopenias por debajo de 50.000 plaquetas en $50 \%$ de los pacientes evaluados (29). En niños cubanos el conteo plaquetario estuvo por debajo de 100.000 por $\mathrm{mm}^{3}$ en el $87 \%$ del grupo con dengue hemorrágico y en el $33 \%$ del grupo con dengue clásico (18).

Llama la atención la frecuencia de coinfecciones en estos casos de dengue, particularmente con malaria, en $17,7 \%(8 / 45)$. Se registraron seis casos con malaria por $P$. vivax y dos casos con 
malaria por $P$. falciparum, hallazgo que sigue siendo repetitivo en los casos registrados después de este estudio (Dirección Local de Salud, municipio de Apartadó. Registros de vigilancia epidemiológica y entomológica, año 2001). Normalmente, los pacientes con malaria no complicada se manejan en forma ambulatoria siempre y cuando no presenten signos de peligro. En este mismo sentido, los pacientes con dengue clásico se supervisan ambulatoriamente, cuando no hay signos de alarma clínicos ni paraclínicos. Sin embargo, el presente estudio nos revela que hubo indicación de manejo hospitalario en los casos de dengue que presentaron infección simultánea por Plasmodium. Ninguno de los casos coinfectados cumplía los criterios de malaria complicada; uno presentó dengue hemorrágico, seis, dengue con manifestaciones hemorrágicas y uno, dengue clásico. Varios estudios muestran que la infección simultánea por dos microorganismos potencia una respuesta inmune de tipo TH1 en el caso de coinfecciones por patógenos intracelulares, exacerbando las manifestaciones clínicas, la gravedad, las complicaciones e, incluso, la mortalidad por estas infecciones. Esta situación ha sido particularmente documentada en el caso de malaria y $\mathrm{VIH}$ (30), malaria y Salmonella typhi, dengue y leptospirosis $(31,32)$, entre otras. En la literatura sólo se ha informado de un caso de coinfección por dengue y malaria en un paciente susceptible que viajó a áreas hiperendémicas para ambas infecciones (33). Otras coinfecciones descritas en el presente estudio fueron infecciones del tracto urinario, bronconeumonía e infección por citomegalovirus. En el estudio de Neiva, las infecciones del árbol traqueo-bronquial fueron las infecciones agregadas más comúnmente observadas (29), lo mismo que en el estudio de dengue hemorrágico en niños de Bucaramanga (8). Así mismo, es importante resaltar la frecuencia importante de dengue en mujeres embarazadas, siendo del 11,1\% (5/45) en el presente estudio.

Vale anotar que en este estudio la letalidad fue elevada $(8,8 \%)$, superando el índice nacional, que fue de $1,2 \%$ en 1998 y de $5,1 \%$ en el 2000 (5), el de América Latina, que fue de 1,3\% en 2002 y los de Perú, Nicaragua y Brasil, con letalidades de
$7,7,7,6$ y $5,6 \%$, respectivamente, considerados como los países de mayor letalidad después de República Dominicana, que alcanza 18,4\% (34). La causa de mayor letalidad en los pacientes con dengue es la presencia del choque circulatorio (35). En el desarrollo de las formas graves del dengue se han involucrado diferentes aspectos, entre ellos, la infección secuencial por más de un serotipo viral, hipótesis conocida como potenciación de la infección dependiente de anticuerpos (36). En los niños menores de un año, la presencia de anticuerpos maternos antidengue transferidos en forma pasiva puede predisponer a la presentación de formas graves por una infección inmunoamplificada (17).

En resumen, el comportamiento clínico del dengue en los pacientes hospitalizados en la Empresa Social del Estado Hospital "Antonio Roldán Betancur" de Apartadó durante el año 2000 se asemeja al comportamiento descrito por otros autores en la literatura, destacándose que hubo mayor gravedad de la enfermedad en niños con edad igual o menor de un año, y la coinfección con otras enfermedades endémicas de la zona, particularmente con malaria.

\section{Agradecimientos}

Los autores del presente trabajo quieren agradecer a Juan Gabriel Piñeros por sus valiosos aportes en la revisión del artículo.

\section{Conflicto de intereses}

No existe conflicto de intereses entre los autores, las instituciones financiadoras y el contenido del presente trabajo.

\section{Financiación}

El presente trabajo fue financiado por el Instituto Colombiano de Medicina Tropical del Instituto de Ciencias de la Salud (ICMT-CES) y la ESE Hospital "Antonio Roldán Betancur" de Apartadó.

\section{Referencias}

1. Organización Panamericana de la Salud. Dengue y dengue hemorrágico en las Américas: guías para su prevención y control. Publicación científica 548. Washington D.C.: OPS; 1995. p.110.

2. Gubler DJ. Dengue and dengue hemorrhagic fever. Clin Microb Rev 1998;11:480-96. 
3. Instituto Nacional de Salud. Ministerio de Salud de la Protección Social. Situación de las enfermedades transmitidas por vectores. SIVIGILA, Febrero 22-28 de 2004, 3-4.

4. Dirección Seccional de Salud de Antioquia. Eventos de Vigilancia epidemiológica, enfermedades transmitidas por vectores y medio ambiente en Antioquia, 1994-2003. [Consultado: marzo 2005]. Disponible en: http://www.dssa.gov.co/vectores/ vectores.htm.

5. Sierra M L, Vélez LM, Castañeda AM, Galeano LA, Molina $A L$, Tabares $Z$, et al. Análisis de la morbimortalidad. Diagnóstico de la situación de salud en Antioquia. Revista Epidemiológica de Antioquia 2000;25:83-205.

6. Restrepo BN, Arboleda M, Lopera T. Estudio seroepidemiológico de dengue en la región del Urabá Antioqueño, Colombia. Infectio 2004;8:255-62.

7. World Health Organization. Dengue and haemorrhagic fever. Diagnosis, treatment, prevention and control. Second edition. Geneva: WHO; 1977. p.84.

8. Méndez A, González G. Dengue hemorrágico en niños: diez años de experiencia clínica. Biomédica 2003;23:180-93.

9. Vargas ME, Aguirre TM, Palacios H. Características clínicas de la fiebre de dengue en niños durante el brote epidémico en Santiago de Cuba. Rev Cubana Med Trop 2001;53:20-3.

10. Rigau-Perez JG, Aayala-Lopez A, Vorndam AV, Clark GG. Dengue activity in Puerto Rico during an interepidemic period (1995-1997). Am J Trop Med Hyg $2001 ; 64: 75-83$

11. Moraes L, Baeta S, Costa M. Encuesta serológica sobre el dengue entre escolares de Río de Janeiro, Brasil, 1986 y 1987. Bol Of Sanit Panam 1991;111:52532.

12. Kouri G, Valdes M, Arguello L, Guzmán MG, Valdes L, Soler M, et al. Epidemia de dengue en Nicaragua, 1985. Rev Inst Med Trop Sao Paulo 1991;33:365-71.

13. Agarwal R, Kapoor S, Nagar R, Misra A, Tandon R, Mathur A, et al. A clinical study of the patients with dengue hemorrhagic fever during the epidemic of 1996 at Lucknow, India. Southeast Asian J Trop Med Public Health 1999;30:735-40.

14. Arunachalam N, Murty US, Kabilan L, Balasubramanian A, Thenmozhi V, Narahari D, et al. Studies on dengue in rural areas of Kurnool District, Andhra Pradesh, India. J Am Mosq Control Assoc 2004;20:87-90.

15. Tewari SC, Thenmozhi V, Katholi CR, Manavalan R, Munirathinam A, Gajanana A. Dengue vector prevalence and virus infection in a rural area in south India. Trop Med Int Health 2004;9:499-07.
16. Mehendale SM, Risbud AR, Rao JA, Banerjee K. Outbreak of dengue fever in rural areas of Parbhani district of Maharashtra (India). Indian $\mathrm{J}$ Med Res 1991;93:6-11.

17. Guha-Sapir D, Schimmer B. Dengue fever: new paradigms for a changing epidemiology. Emerg Themes Epidemiol 2005;2:1.

18. Martínez E, Guzmán MG, Valdés M, Soler M, Kouri G. Fiebre del dengue y dengue hemorrágico en infantes con infección primaria. Rev Cubana Med Trop 1993; 45:97-101.

19. Aguirre C. Aspectos clínicos del dengue. Infectio 2004;8:220-4.

20. Convers SM, Villar LA, Martínez RA, Méndez CX, Gómez JA, Rojas EM. Clínica gastrointestinal y su asociación con la severidad en dengue. Infectio 2001;5:21-30.

21. Guzmán MG, Kouri G, Bravo J, Colunga M, Soler $\mathbf{M}$, Vasques $\mathbf{S}$, et al. Dengue haemorrhagic fever in Cuba II. Clinical investigation. Trans R Soc Trop Med Hyg 1984;78:239-41.

22. Rosso F, Restrepo MT, Alzate A, Munoz, Moreno CH. Dengue hemorrágico en el Hospital Universitario del Valle, 1990-1992. Colombia Médica 1994;25:10-4.

23. Díaz FJ. Fiebre amarilla, dengue y encefalitis por arbovirus. En: Restrepo A, Robledo R, Leiderman E, Restrepo M, Botero D, Bedoya V, editores. Fundamentos de medicina. Enfermedades infecciosas. 6aㅡ. Medellín: Corporación para Investigaciones Biológicas; 2003. p.787-91.

24. Chanarin I, Walford DM. Thrombocytopenic purpura in cytomegalovirus mononucleosis. Lancet 1973;2:238 9

25. Crumpacker CS. Cytomegalovirus, Chapter 127. En: Mandell GL, Dolin R, Bennett JE. Principles and practices of infectious diseases. Fifth Edition. New York: Churcill Livingstone; 2000. p.3263.

26. World Health Organization. Severe $P$. falciparum malaria. Trans R Soc Trop Med Hyg 2000;94(Suppl. 1):1-90.

27. Gonzalez MG, Carmona J F, Lopera T, Blair S. Características clínico epidemiológicas de 291 pacientes hospitalizados por malaria en Medellín (Colombia). Acta Médica Colombiana 2000;25:16370 .

28. Restrepo BN, Isaza DM, Salazar CL, Upegui GE, Duque CL, Ospina M, et al. Efectos del virus de dengue durante el embarazo. Infectio 2002;6:197-203.

29. Salgado D, Rodríguez A, Vega R. Dengue hemorrágico. Emergencia pediátrica en el Huila. Pediatria 1999;34 (2). [Consultado: marzo 2004]. Disponible en: http://www.encolombia.com/pediatria 34299 dengue6.htm. 
30. World Health Organization. Malaria and HIV interactions and their implications for public health policy. Report of a technical consultation. Geneva: WHO; 2004. [Consultado: abril de 2006]. Disponible en:www.who.int/malaria/malaria_HIV/ MalariaHIVinteractions_report.pdf.

31. Levett PN, Branco SL, Edwards CN. Detection of dengue infection in patients investigated for leptospirosis. Am J Trop Med Hyg 2000;62:112-4.

32. Flannery B, Pereira MM, Velloso LD, Carvalho CD, De Codes LG, Orrico GD, et al. Referral pattern of leptospirosis cases during a large urban epidemic of dengue. Am J Trop Med Hyg 2001;65:657-63.

33. Charrel RN, Brouqui P, Foucault C, d Lamballerie X. Concurrent dengue and malaria. Emerg Infect Dis 2005;11:1153-4.
34. Pan American Health Organization. Number of reported cases of dengue \& dengue hemorrhagic fever region of Americas (by country and subregion). OPS. 2002. [Consultado: junio de 2003]. Disponible en: http:/ /www.paho.org/English/ad/dpc/cd/dengue-cases2002.htm.

35. Guzman MG, Kouri G, Morier L, Soler M, Fernández A. A study of fatal hemorrhagic dengue cases in Cuba, 1981. Bull Pan Am Health Organ 1984;18: 213-20.

36. Halstead SB. The pathogenesis of dengue. Molecular epidemiology in infections disease. Am J Epidemiol 1981;114:632-48.

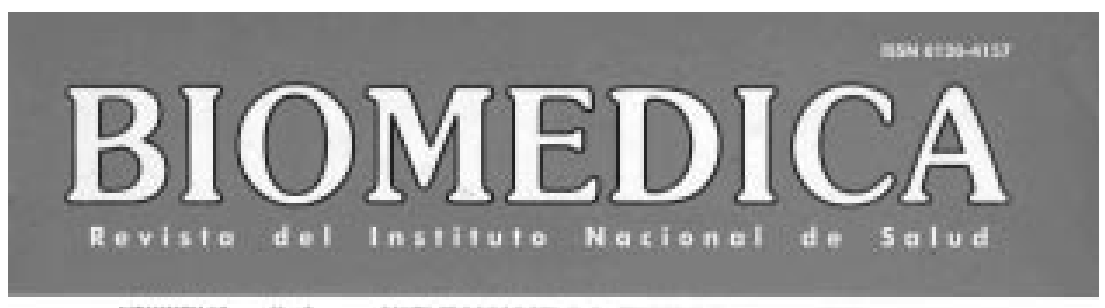

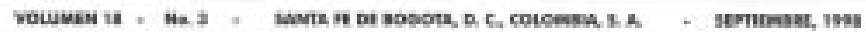

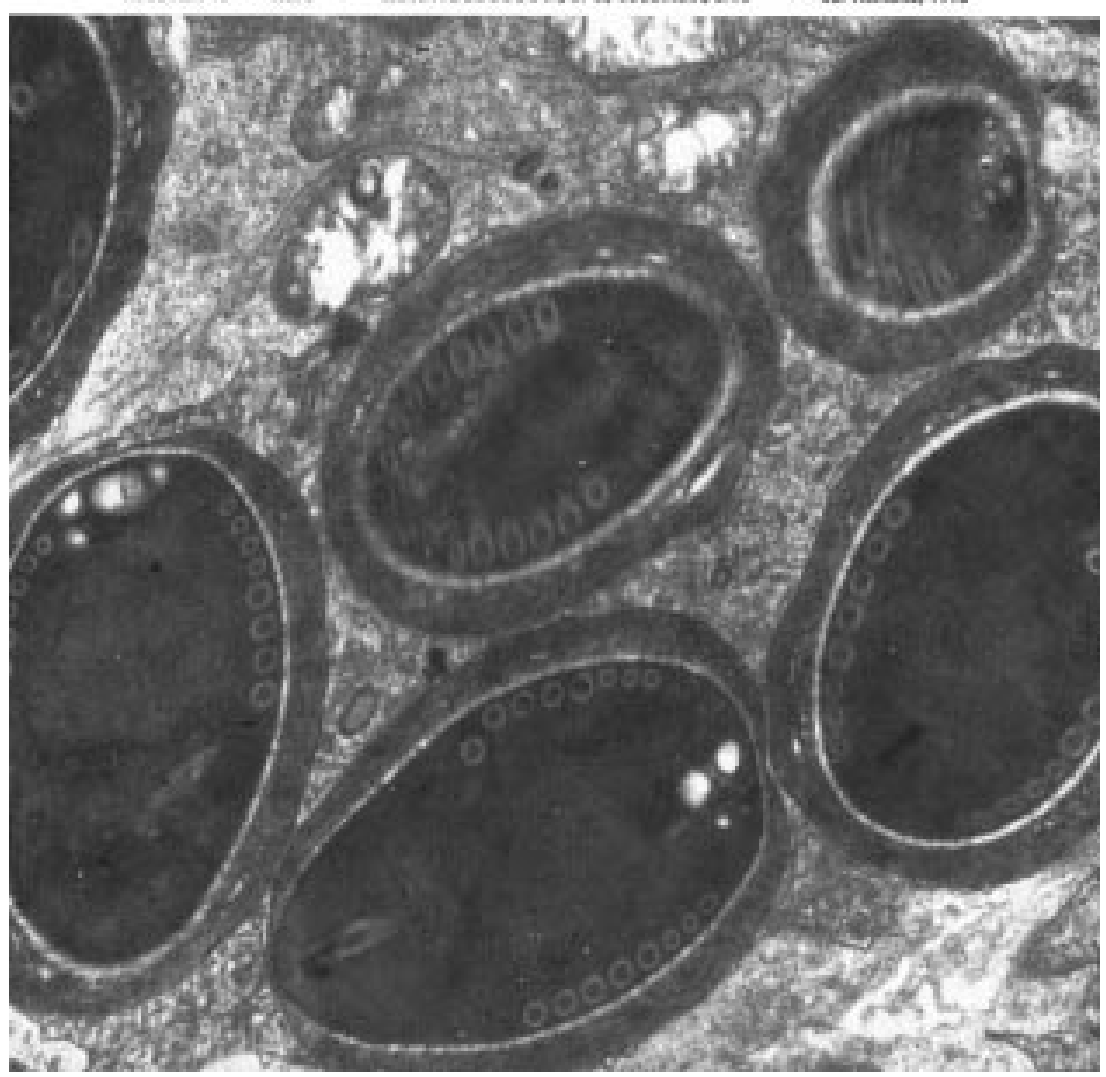

